\title{
Influence of Temperature and Humidity on Carbon Based Printed Flexible Sensors
}

\author{
Anindya Nag, Subhas Mukhopadhyay \\ Faculty of Science and Engineering \\ Macquarie University \\ Sydney, Australia \\ Email: anindya1991@gmail.com
}

\author{
Jurgen Kosel \\ Computer, Electrical and Mathematical Sciences and \\ Engineering \\ King Abdullah University of Science and Technology \\ Thuwal, Saudi Arabia
}

\begin{abstract}
This paper presents the response of two different types of novel printed sensors towards the change in temperature and humidity. The electrodes of all the sensors were based on carbon materials. Followed by the design and fabrication of the sensors, the responses of the sensors were analyzed for different temperature and humidity conditions in an incubator. These results provide a podium to enhance the alternation of the fabrication procedure of carbon-based printed sensors.
\end{abstract}

Keywords- Carbon Nanotube; PDMS; Graphene; Polyimide; Temperature; Humidity.

\section{INTRODUCTION}

After the involvement of sensors in different day to day applications, researchers all over the world started to optimize the drawbacks involved in the production of sensing systems to generate them on a larger scale. The techniques for design and fabrication of different kinds of sensors have continuously evolved with technology and time. Initially, when the sensors got introduced commercially around two decades back [1, 2] to be used for different applications, silicon-based sensors where one of the most popular choices [3-5] at that time due to certain advantages. Some of them are the easy implementation, its compactness and power-saving phenomenon it had compared to the devices used prior its introduction. Eventually, with the betterment of technology, a lot of fabrication techniques and processing materials got introduced which had their characteristics much better when compared to the silicon sensors. A flexible sensor is one sector that has revolutionized the sensory world in terms of performance and cost required to develop the system [6]. The flexible materials used as the raw material during the fabrication differ on the basis of Young's modulus (E), electrical, mechanical and thermal conductivity. In today's world, the flexibility of almost every material has been introduced in terms of the nanoparticle. Electrical conductivity, mechanical flexibility and operating range of temperature and humidity are some of the primary features of material studied before it is chosen to develop the electrodes. Carbon [7], silver [8], aluminum [9], gold [10], and zinc [11] are some of the common elements used as nanoparticles for developing electrodes. Among them, carbon [12] can be attributed to the very popular choice because of its certain advantages like lightweight, very high electrical conductivity and mechanical flexibility. One more reason that can be added to these attributes is its corrosion resistant nature [13]. This makes it a much more popular choice for developing the electrodes compared to other metals. The second part of the sensor comprises of the substrate of the sensor. This part stands equally crucial, especially for the flexible sensors because even though the response of the sensor is collected from the electrodes, the substrates are the locations of impact. Among the different materials used to develop the substrate, Polydimethylsiloxane (PDMS) [14], Polyethylene terephthalate (PET) [15], Polyethylene naphthalate (PEN) [16], Polydiallyldimethylammonium chloride (PDDA) [17], and Polyimide (PI) [18] are some of the common ones used to fabricate them. The third category involved with the fabrication process is the technique with which the prototypes are made. Photolithography [19], screen printing [20], ink-jet printing [21], 3-D printing [22], vapor deposition processes $[23,24]$, coating processes [25, 26], and laser cutting [27] are some of the common techniques. Among these, laser cutting [28-30] was used to develop some flexible sensors due to some of the advantages like easy sample preparation, no post processing step, and smooth cut edges. One of the primary steps involved in the development of a sensor is its design and fabrication. This is decided by a number of factors like the cost of the processing material, the fabrication technique, the application involved with the developed sensor, etc. Among these factors, the application is the main one on which the idea behind the design of the sensor depends a lot. The application of a sensor decides the ambiance the sensor would be used for monitoring purposes. Temperature and humidity are the two important factors that are studied very closely during the fabrication of a sensor. This is because, due to the high sensitivity of the currently available sensors, the variation in the environmental conditions can cause a significant change in the response of the sensor. There are large variations in the range of sensors with respect to their operating temperature and humidity. Even slight changes from the range can high large erroneous results. So, while designing the sensor, the temperature and humidity range is carefully studied to determine their optimal range. The paper presents the fabrication of some of the novel carbon-based sensors and 
their response to different temperature and humidity conditions. The results shown in this paper can be attributed to the specific range of frequencies to which the sensors are sensitive with respect to the change in the range of temperature and humidity.

The paper is subdivided into four sections. After the introduction given in section I, the fabrication of the two types of sensor patches is explained in section II. Section III gives the experimental results of the temperature and humidity testing of the fabricated sensors. The discussions related to the results and the conclusion to the paper is given in the final section.

\section{FABRICATION OF THE SENSOR PATCHES}

The development of all the sensor patches was done in the laboratory conditions. The electrodes of each of the prototypes were developed interdigitated due to certain reasons. Among them, the increase in both effective sensing area capacitance and the reduction in self-resonant frequency are some of the main reasons. The first sensor patch was fabricated by the formation of nanocomposite to develop the electrodes. Figures 1(a)-1(d) show the schematic diagram of the first sensor prototype formation [30]. The base elastomer and curing agent of PDMS (SYLGARD ® 184, Silicon Elastomer) were mixed at a ratio of 10:1 to form the PDMS on a Poly (methyl methacrylate) PMMA template. PMMA was considered as a template mainly due to three important reasons of its nointeraction with the cured PDMS, easy peeling off the PDMS and better adherence with the cured PDMS. The height of the casted PDMS was adjusted with a casting knife (SHEEN, $1117 / 1000 \mathrm{~mm}$ ) to around $1 \mathrm{~mm}$. Then, followed by the desiccation of the sample for 2 hours to remove any trapped air bubbles, it was cured in an oven for 8 hours at $80^{\circ} \mathrm{C}$. A nanocomposite (NC) layer comprising of PDMS and MWNCTs (Aldrich, 773840-100G) was cast on top of the PDMS layer. The mixture of MWCNTs and PDMS to form the NC was optimized to $4 \mathrm{wt}$. $\%$ of former in the later. A trade-off was done between the flexibility and conductivity to determine the wt. \%. Figure 2 shows the top-view of the SEM image of the NC. The white spots in the figure represent the PDMS whereas the black spots represent the agglomeration of the MWCNTs and PDMS.

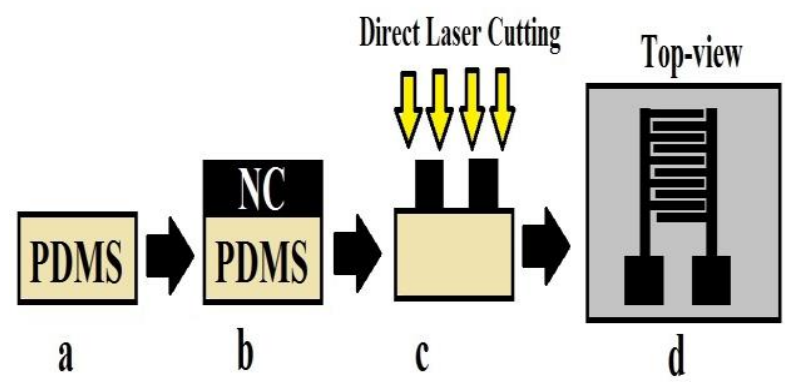

Fig. 1: Schematic diagram of the fabrication of CNT-PDMS based sensor patch. (a) The casting and curing of PDMS were done to develop the substrate of the sensor. (b) A layer of nanocomposite (NC) was formed on top of the PDMS to develop the electrodes. (c) Laser cutting on the NC layer was done to form the electrodes. (d) The top-view of the sensor patch.

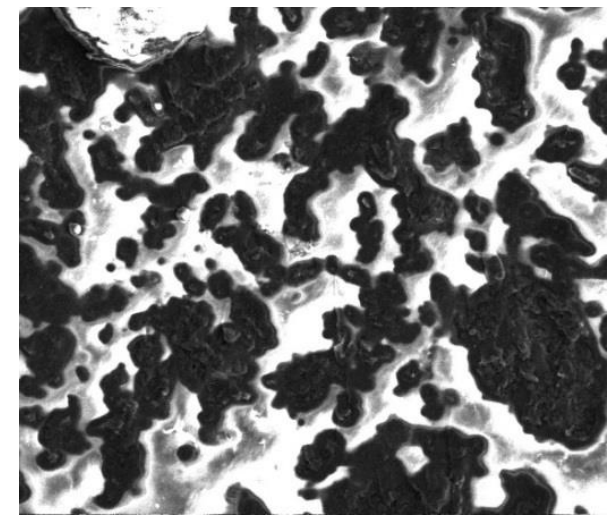

Fig. 2: SEM image of the nanocomposite of 4 wt. \% of CNTs in PDMS

The height of the casted NC was adjusted to around 600 microns by the casting knife. Similar to curing of the PDMS, the sample was first desiccated and cured for the same duration to form the cured NC layer. Finally, the template was taken for laser cutting to develop the electrodes on the NC layer. The dimensions of the electrodes were specified in a drawing software (CorelDraw) attached to the laser system (Universal Laser Systems). Laser cutting was done by scanning off the residual NC from the top layer leaving just the electrodes. In the process of laser cutting of the electrodes, power $(\mathrm{W})$, speed $(\mathrm{m} / \mathrm{min})$ and $\mathrm{z}$-axis $(\mathrm{mm})$ are the three parameters of the laser systems that were tuned to achieve optimized electrodes. Power can be defined as the amount of energy the laser contains while striking the sample. Speed refers to the movement of the laser nozzle over the sample. Zaxis defines the height of the laser platform to adjust the focal point of the laser on the sample.

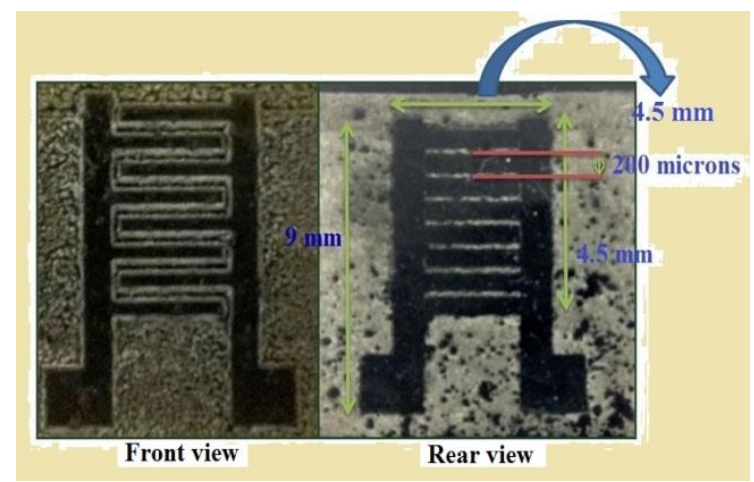

Fig. 3: Front and back view of the final sensor patch developed with CNT and PDMS.

After a series of experiments done with the tuning of the parameters and short circuitry tests of the after product, a combination of $24 \mathrm{~W}, 70 \mathrm{~m} / \mathrm{min}$ and $1 \mathrm{~mm}$ as the power, speed, and z-axis respectively, was chosen as the viable one. Figure 3 shows the front and rear view of the final product. Four pairs of electrode fingers were formed with a thickness of 200 microns of each finger. The interdigital distance (d) was 100 microns with a surface area of $20.25 \mathrm{~mm}^{2}$ of the sensing area of the patch. Due to the flexible nature of these 
sensors, they were later used for monitoring limb movements and respiration [30]. The sensor patches were attached to the joints with biocompatible tapes to the limbs of a person to detect its movement. For monitoring of respiration, the sensor patch was attached to the lower part of the diaphragm to utilize the flexible nature of the sensor in detecting the inhalation and exhalation during respiration.

The second type of carbon based sensor was developed from the same material but with a different technique. These sensor prototypes were fabricated with casting method [29]. Figure 4 shows the schematic diagram of the individual fabrication steps of the sensor patches. Similar to the first type of sensor, PMMA was considered as a template to develop the sensor because of its mentioned advantages. Laser cutting was done on the template to create trenches. The dimensions of the trenches defined the dimensions of the electrodes that were to be developed. As the height of the tranches would have defined the height of the electrodes, the laser parameters (power, speed, and z-axis) were optimized with a series of experiments. Finally, they were fixed to $15 \mathrm{~W}, 45 \mathrm{~m} / \mathrm{min}$ and $1 \mathrm{~mm}$ for the power, speed and $\mathrm{z}$-axis respectively. After developing the trenches on the PMMA template, a layer of NC was cast on the template. The residual $\mathrm{NC}$, leaving the parts inside the trenches was scrapped off the sample. This was desiccated followed by its curing at $80^{\circ} \mathrm{C}$ for 8 hours.

The second type of carbon based sensor involved the generation of conductive material from polymer films [31]. Laser writing was done on commercial PI films to induce graphene. The $\mathrm{sp}^{3}$ hybridized carbon atoms in the PI films were photo-thermally converted to $\mathrm{sp}^{2}$ hybridized carbon atoms in graphene. This conductive material was then used as electrodes in a sensor patch. Figures 6(a)-6(d) show the schematic diagram of the fabrication procedure. Initially, the PI film was fixed to a glass template and was taken for laser writing purposes. After the dimensions had been defined in the laser cutting system, induced graphene was generated from the polymer films. Figure 7 depcits the SEM image of the transferred graphene on the Kapton tapes. It is seen that the transferred graphene has come off almost cleanly on the Kapton tapes. The electrode lines are also perpendicular and smooth which nullifies the requirement of post-processing step for the sensor patch. The laser parameters (power, speed, and $\mathrm{Z}$-axis) optimized for this process were power- $9 \mathrm{~W}$, speed- 70 $\mathrm{m} / \mathrm{min}$ and $\mathrm{z}$-axis- $1 \mathrm{~mm}$. The induced graphene was then transferred to Kapton tapes to use as electrodes in a sensor. The Kapton tapes were stuck on the graphene followed by applying manual pressure to it. Once the graphene was transferred to the tape, it was carefully pulled off the substrate PI film to avoid the damage on the electrodes. These were $<20$ $\mathrm{mS} / \mathrm{m}$ difference in the conductivity between the induced and transferred graphene. Figure 7 shows the final product which was used as a sensor patch. The prototype consisted of six pairs of electrode fingers with each finger having a length and width of 500 microns and 100 microns respectively. The total sensing area was $96 \mathrm{~mm}^{2}$. These sensor patches are largely favorable to be used for different applications due to the high conductivity of the electrodes.

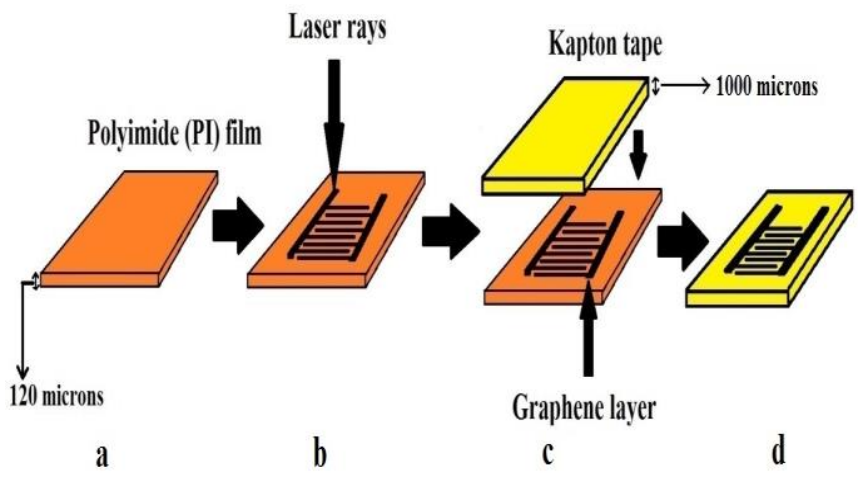

Fig. 6: Schematic diagram of the fabrication steps of the sensor patch develop with graphene and PI. (a) The PI film was attached to the glass substrate for laser ablation. (b) Photo-thermally induced graphene was formed from the PI film. (c) The formed graphene was transferred to Kapton tapes by sticking and the tapes on the graphene. (d) The transferred graphene was on the tapes was used a sensor patch.

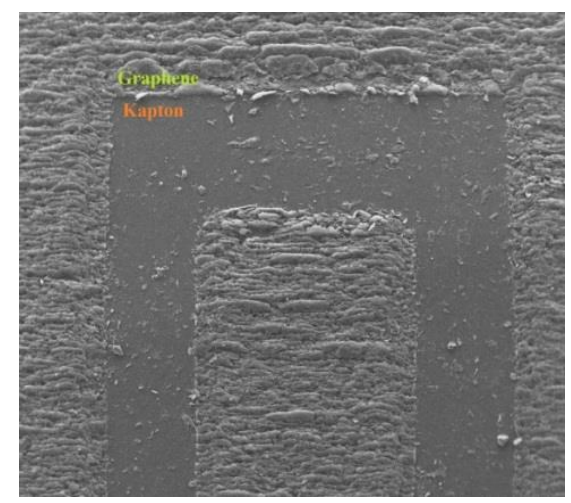

Fig. 7: SEM image of the top-view of the transferred graphene on the Kapton tapes.

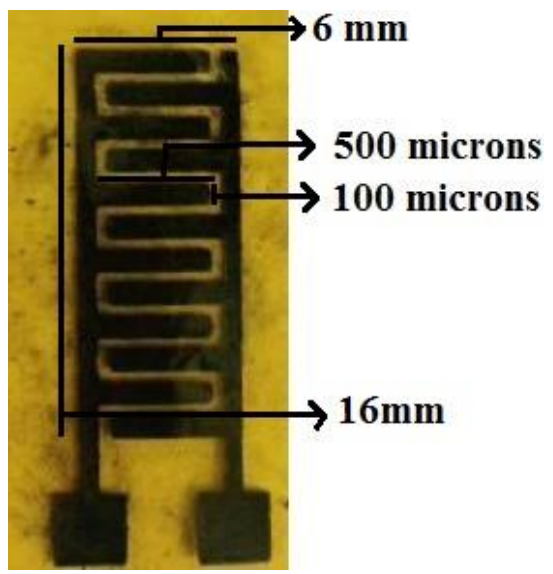

Fig. 8: Final sensor patch developed with graphene and PI.

\section{WORKING PRINCIPLE OF THE ELECTRODES}

The interdigitated electrodes work on the principle of capacitive sensing. The design of the electrodes resembles that of a parallel plate capacitor, but in a planar way. This helps in non-invasive, one-sided measurement of dielectric materials. 
Figure 9 shows the schematic for the working principle of the sensors. The two electrodes are referred to as the excitation and sensing electrodes. Due to its planar nature of the electrodes, when a small time dependent voltage is applied to the electrodes, the electric field bulges from one electrode to another of opposite polarity. If any material is kept in contact or proximity to the sensing area of the patch, the electric field penetrates which traveling from one electrode to another. The properties of this field are studied in order to study the properties of the sample under test.

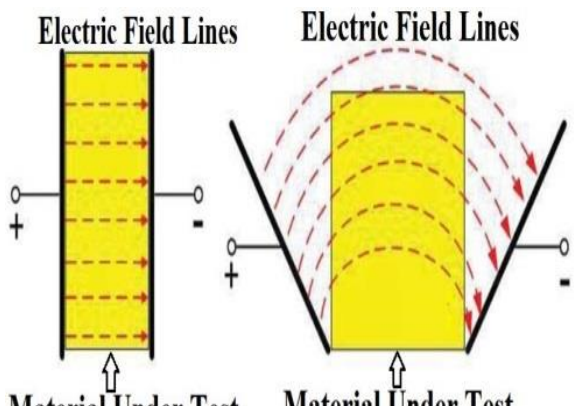

Material Under Test Material Under Test b

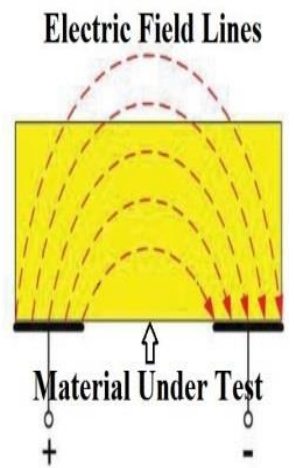

C

Fig. 9: Schematic diagram showing the working principle of the interdigital sensors. (a) The sensor works on the idea of a parallel plate capacitor. (b) The interdigital sensor represents the electrodes in a planar way. (c) With the application of voltage, the electric field bulges from one electrode to another of opposite polarity [32].

\section{EXPERIMENTAL RESULTS}

The experiments were performed in same laboratory conditions as that followed during the fabrication of the sensor patches. An incubator (Heraeus, Thermo Fisher Scientific) was used to perform the temperature and humidity variations. Figure 10 shows the schematic diagram regarding the collection of the data from the sensor. While the sensor was placed in the incubator with defined conditions on the instrument, a high tester HIOKI IM 3536 LCR Hi precision tester was connected to the sensor from another side through Kelvin probes for data analysis. The LCR meter was connected to a laptop via USB port for data collection in an excel file using automatic data acquisition algorithm. A sinusoidal voltage signal of 1 Volt peak-to-peak was supplied from the LCR meter to the excitation electrode of the sensor. A frequency sweep between $1 \mathrm{~Hz}$ to $100 \mathrm{kHz}$ was performed to determine the response of the sensor patches over this range at different temperature and humidity conditions. The responses of the sensors were analyzed in terms of phase angle with respect to frequency. Phase angle can be defined as the angle between the input voltage and output current. Figures 11 and 12 show the responses of the two sensor patches towards the variation in temperature. Three different temperature values $\left(30^{\circ} \mathrm{C}-40^{\circ} \mathrm{C}\right)$ with a difference of $5^{\circ} \mathrm{C}$ were tested with the sensor patches. The sensitive regions towards different temperature values for each sensor are PDMS-CNT: $57.5 \mathrm{kHz}$ $-100 \mathrm{kHz}$, graphene: $53.5 \mathrm{kHz}-100 \mathrm{kHz}$. It is seen from the figures that these two sensors are sensitive towards highfrequency ranges.

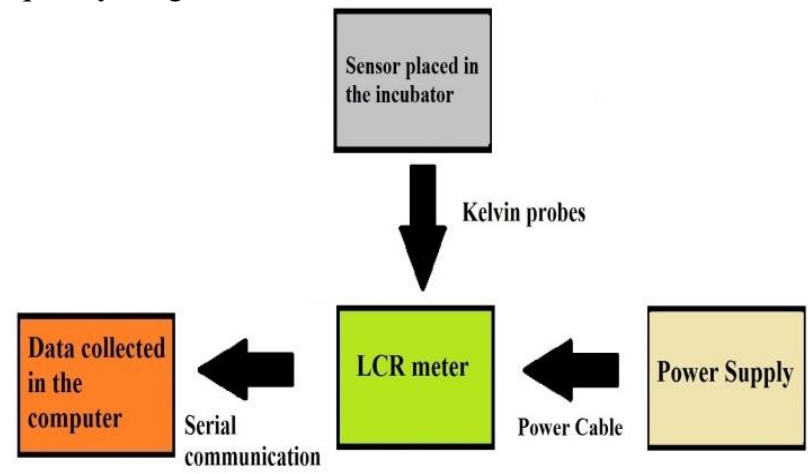

Fig. 10: Connection of the sensor to the LCR for data collection.

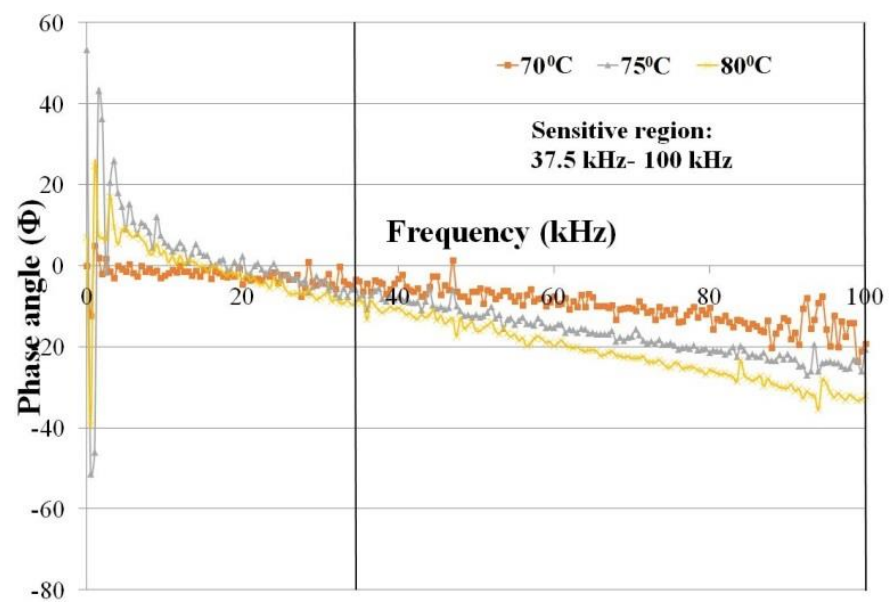

Fig. 11: Response of the PDMS- CNT-based sensor towards the variation in temperature with respect to frequency.

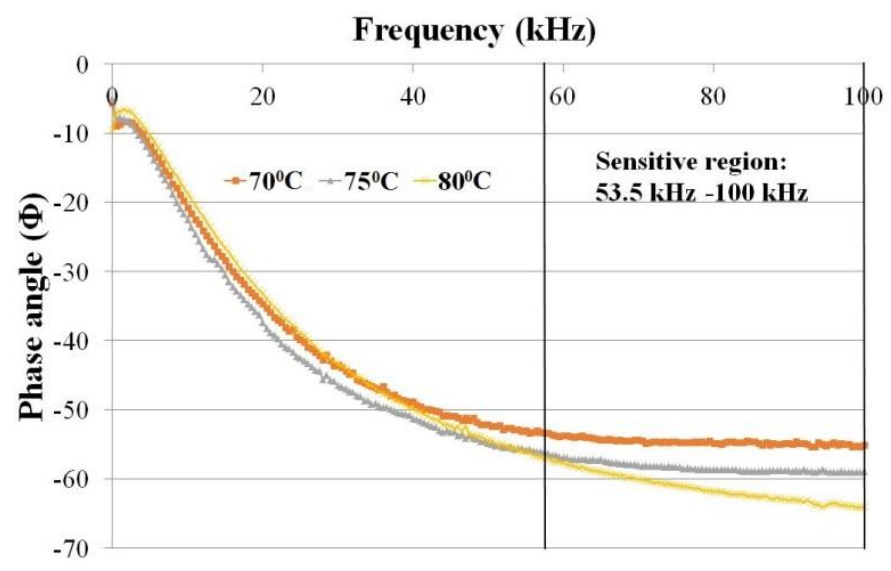

Fig. 12: Response of the graphene sensor towards the variation in temperature with respect to frequency.

For the response of the sensor patches towards the change in humidity, three different humidity values $(60 \% \mathrm{RH}-70 \% \mathrm{RH})$ with a difference of $5 \% \mathrm{RH}$ were considered for experimentation. Since the normal humidity value in the laboratory environment would lie between $40 \% \mathrm{RH}-50 \%$ $\mathrm{RH}$, it was made sure that the chosen values for the 
experiments would be different from them. This could increase the potentiality of the sensors for different applications. Figures 14 and 15 show the responses of the CNT-PDMS and graphene sensors. Even though the frequency sweep was done between $1 \mathrm{~Hz}-100 \mathrm{kHz}$, only a specific region of frequency is shown in the results to identify the differences. The sensitive regions for the different values of relative humidity for the CNT-PDMS and graphene sensor lie between $612.41 \mathrm{~Hz}-913.62 \mathrm{~Hz}$ and $359.65 \mathrm{~Hz}-959.05 \mathrm{~Hz}$. It is seen that the sensitive frequency regions for both the sensors for the change in temperature are in a completely different range from that of the change in humidity. The sensors operate at higher frequencies for the change in temperature while for humidity; they work in lower frequency values. This is plausible as the increase in temperature reduces the amount of water vapor present inside the incubator causing a decrease in capacitance [33].

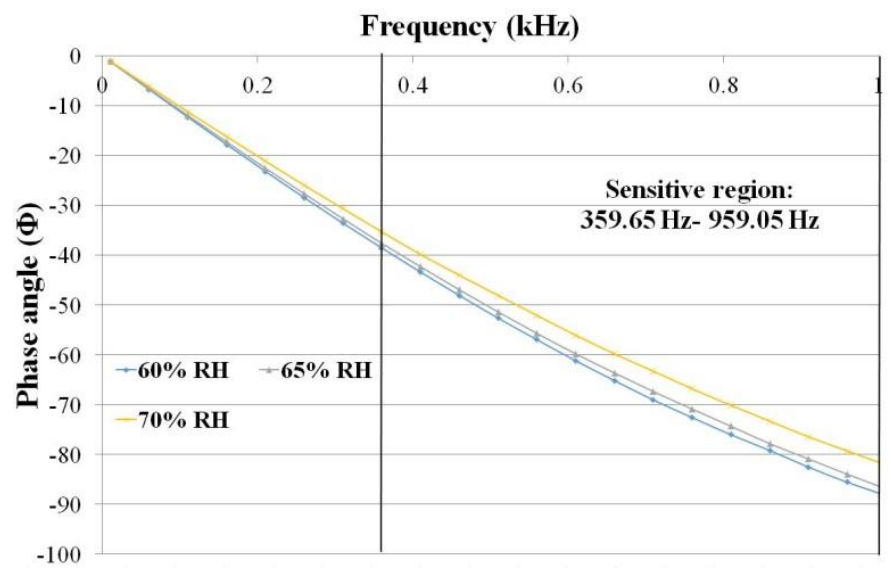

Fig. 13: Response of the PDMS-CNT sensor towards the variation in humidity with respect to frequency.

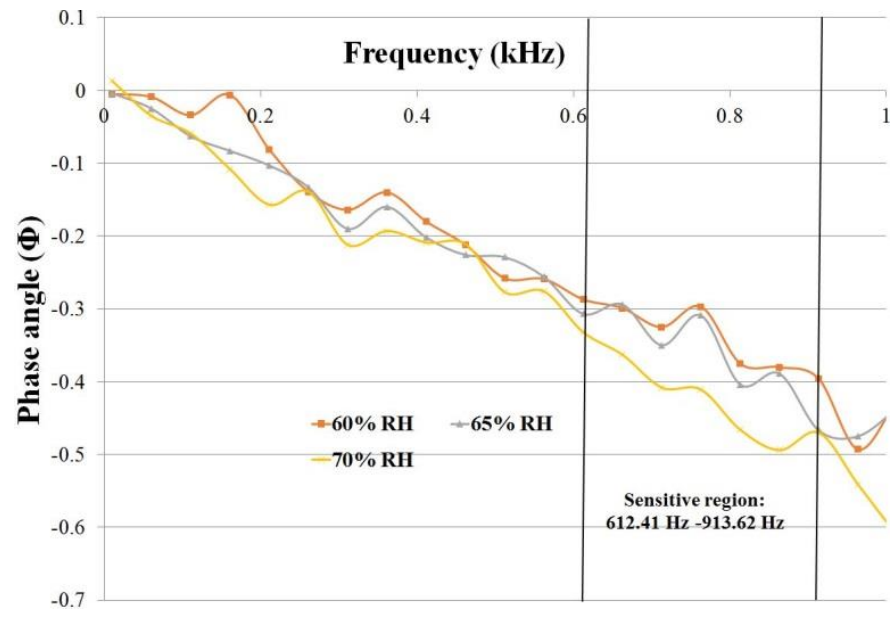

Fig. 14: Response of the graphene sensor towards the variation in humidity with respect to frequency.

\section{CONCLUSION}

The paper reported the response of the two novel printed sensors towards the variation in temperature and humidity. The above experimental results show that the sensors have the potential to be used for applications demanding ambiance conditions that are different from the normal laboratory settings. Further characterization of these fabricated sensors would be done in future to have a better understanding of these sensors to use them for different applications.

\section{ACKNOWLEDGMENT}

The authors would like to thank King Abdullah University of Science and Technology (KAUST), Saudi Arabia to provide the research facilities to fabricate the sensor patches. They would also like to thank Macquarie University for providing the opportunities to test the sensor prototypes.

\section{REFERENCES}

9]

A. Nag, and S. Mukhopadhyay, "Smart Home: Recognition of activities of elderly for 24/7; Coverage issues." pp. 480-489 S. M. Sze, Semiconductor sensors: Wiley New York, 1994.

A. Nag, A. I. Zia, X. Li et al., "Novel Sensing Approach for LPG Leakage Detection: Part I-Operating Mechanism and Preliminary Results," IEEE Sensors Journal, vol. 16, no. 4, pp. 996-1003, 2016.

S. C. Mukhopadhyay, and C. P. Gooneratne, "A novel planar-type biosensor for noninvasive meat inspection," IEEE Sensors Journal, vol. 7, no. 9, pp. 1340-1346, 2007.

A. I. Zia, M. S. A. Rahman, S. C. Mukhopadhyay et al., "Technique for rapid detection of phthalates in water and beverages," Journal of Food Engineering, vol. 116, no. 2, pp. 515523, 2013.

F. Jiang, Y.-C. Tai, K. Walsh et al., "A flexible MEMS technology and its first application to shear stress sensor skin." pp. 465-470. M. Amjadi, and I. Park, "Carbon nanotubes-ecoflex nanocomposite for strain sensing with ultra-high stretchability." pp. 744-747.

J. Lee, J. Y. Woo, J. T. Kim et al., "Hybrid Transparent Electrodes of Silver Nanowires and Carbon Nanotubes by Plasmonic Welding," 대한기계학회 2014 년도 추계학술대회, pp. 27792784, 2014.

W. Hu, S. N. Zhang, X. Niu et al., "An aluminum nanoparticleacrylate copolymer nanocomposite as a dielectric elastomer with a high dielectric constant," Journal of Materials Chemistry C, vol. 2, no. 9, pp. 1658-1666, 2014.

H. HyeáJang, and M. SuáHan, "A functionalized gold nanoparticles-assisted universal carrier for antisense DNA," Chemical Communications, vol. 46, no. 23, pp. 4151-4153, 2010. E. S. Ates, S. Kucukyildiz, and H. E. Unalan, "Zinc oxide nanowire photodetectors with single-walled carbon nanotube thinfilm electrodes," ACS applied materials \& interfaces, vol. 4, no 10, pp. 5142-5146, 2012

J. Bai, C. Sun, and X. Jiang, "Carbon dots-decorated multiwalled carbon nanotubes nanocomposites as a high-performance electrochemical sensor for detection of $\mathrm{H} 2 \mathrm{O} 2$ in living cells," Analytical and bioanalytical chemistry, vol. 408, no. 17, pp. 47054714, 2016

[13] K. J. Loh, J. Kim, J. P. Lynch et al., "Multifunctional layer-bylayer carbon nanotube-polyelectrolyte thin films for strain and corrosion sensing," Smart Materials and Structures, vol. 16, no. 2, pp. 429, 2007.

[14] B.-H. Jo, L. M. Van Lerberghe, K. M. Motsegood et al., "Threedimensional micro-channel fabrication in polydimethylsiloxane (PDMS) elastomer," Journal of microelectromechanical systems, vol. 9, no. 1, pp. 76-81, 2000.

[15] M.-x. Jing, C. Han, M. Li et al., "High performance of carbon nanotubes/silver nanowires-PET hybrid flexible transparent 
conductive films via facile pressing-transfer technique," Nanoscale research letters, vol. 9, no. 1, pp. 1-7, 2014.

[16] R. Shabannia, and H. Abu-Hassan, "Vertically aligned ZnO nanorods synthesized using chemical bath deposition method on seed-layer $\mathrm{ZnO} /$ polyethylene naphthalate (PEN) substrates,' Materials Letters, vol. 90, pp. 156-158, 2013.

[17] Y. Wang, Z. Yang, Z. Hou et al., "Flexible gas sensors with assembled carbon nanotube thin films for DMMP vapor detection," Sensors and Actuators B: Chemical, vol. 150, no. 2, pp 708-714, 2010.

[18] Y. Qin, Q. Peng, Y. Ding et al., "Lightweight, superelastic, and mechanically flexible graphene/polyimide nanocomposite foam for strain sensor application," ACS nano, vol. 9, no. 9, pp. 8933-8941, 2015 .

[19] S. Khumpuang, H. Maekawa, and S. Hara, "Photolithography for minimal fab system," IEEJ Transactions on sensors and micromachines, vol. 133, no. 9, pp. 272-277, 2013.

[20] S. Ohta, S. Komagata, J. Seki et al., "All-solid-state lithium ion battery using garnet-type oxide and Li 3 BO 3 solid electrolytes fabricated by screen-printing," Journal of Power Sources, vol. 238, pp. 53-56, 2013.

[21] C. M. Homenick, R. James, G. P. Lopinski et al., "Fully Printed and Encapsulated SWCNT-Based Thin Film Transistors via a Combination of R2R Gravure and Inkjet Printing," ACS applied materials \& interfaces, vol. 8, no. 41, pp. 27900-27910, 2016.

[22] G. Comina, A. Suska, and D. Filippini, "PDMS lab-on-a-chip fabrication using 3D printed templates," Lab on a Chip, vol. 14, no. 2, pp. 424-430, 2014.

[23] K. Parikh, K. Cattanach, R. Rao et al., "Flexible vapour sensors using single walled carbon nanotubes," Sensors and Actuators B: Chemical, vol. 113, no. 1, pp. 55-63, 2006.

[24] Z. C. Kennedy, J. F. Christ, K. A. Evans et al., "3D-printed poly (vinylidene fluoride)/carbon nanotube composites as a tunable, low-cost chemical vapour sensing platform," Nanoscale, vol. 9, no. 17, pp. 5458-5466, 2017.

[25] A. Nag, A. I. Zia, X. Li et al., "Novel Sensing Approach for LPG Leakage Detection-Part II: Effects of Particle Size, Composition, and Coating Layer Thickness," IEEE Sensors Journal, vol. 16, no. 4, pp. 1088-1094, 2016.

[26] K. Ahmed, N. Yamada, M. Wada et al., "Hydrogel Coating on Soft Polymeric Substrates for Microfabricated Devices." pp. 38623862 .

[27] Z. Isiksacan, M. T. Guler, B. Aydogdu et al., "Rapid fabrication of microfluidic PDMS devices from reusable PDMS molds using laser ablation," Journal of Micromechanics and Microengineering, vol. 26, no. 3, pp. 035008, 2016.

[28] A. Nag, S. C. Mukhopadhyay, and J. Kosel, "Tactile Sensing From Laser-Ablated Metallized PET Films," IEEE Sensors Journal, vol. 17, no. 1, pp. 7-13, 2016.

[29] A. Nag, S. Mukhopadhyay, and J. Kosel, "Transparent biocompatible sensor patches for touch sensitive prosthetic limbs." pp. 1-6.

[30] A. Nag, S. C. Mukhopadhyay, and J. Kosel, "Flexible carbon nanotube nanocomposite sensor for multiple physiological parameter monitoring," Sensors and Actuators A: Physical, vol. 251, pp. 148-155, 2016.

[31] J. Lin, Z. Peng, Y. Liu et al., "Laser-induced porous graphene films from commercial polymers," Nature communications, vol. 5, 2014.

[32] M. S. A. Rahman, S. C. Mukhopadhyay, and P.-L. Yu, "Novel Planar Interdigital Sensors," Novel Sensors for Food Inspection: Modelling, Fabrication and Experimentation, pp. 11-35: Springer, 2014.

[33] P. Mruetusatorn, G. Polizos, P. G. Datskos et al., "Control of membrane permeability in air-stable droplet interface bilayers," Langmuir, vol. 31, no. 14, pp. 4224-4231, 2015. 